\title{
Costs of predicting IDDM
}

\author{
J. Hahl ${ }^{1,2}$, T. Simell ${ }^{2}$, J. Ilonen ${ }^{3}$, M. Knip ${ }^{4,5}$, O. Simell ${ }^{2}$ \\ ${ }^{1}$ Department of Economics, Turku School of Economics and Business Administration, Turku, Finland \\ ${ }^{2}$ Department of Pediatrics, University of Turku, Turku, Finland \\ ${ }^{3}$ Department of Virology, University of Turku, Turku, Finland \\ ${ }^{4}$ Department of Pediatrics, University of Oulu, Oulu, Finland \\ ${ }^{5}$ Department of Pediatrics, Tampere University Hospital, Tampere, Finland
}

Summary Programmes aiming at prediction and prevention of insulin-dependent diabetes mellitus (IDDM), a multifactorial autoimmune disease, have been launched or are in the planning phase in several countries. We hypothesized that the costs of finding the correct target subjects for preventive interventions are likely to vary markedly according to the prediction strategy chosen. Average direct costs accruing in the Finnish IDDM Prediction and Prevention Project (DIPP) were analysed from the health care provider's viewpoint. The genetically targeted strategy included costs of assessing genetic IDDM susceptibility followed by measurement of marker(s) of islet autoimmunity in the susceptibility restricted population at 3 to 6 -month intervals. In the pure immunological strategy markers of autoimmunity were repeatedly analysed in the entire population. The data were finally exposed to sensitivity analysis. The genetically targeted prediction strategy is cost-saving in the first year if autoimmune markers are analysed as frequently as under the DIPP project, and in all circumstances later. The 10-year direct costs per child are US\$ 245 (present value \$217, 5\% discount rate) if the genetically targeted approach is used and $\$ 733$ (present value \$ 619) if the pure immunological strategy is chosen. In sensitivity analysis the 10 -year costs (present value) per child of the genetically targeted strategy and of the pure immunological strategy varied from $\$ 152$ to $\$ 241$ and from $\$ 430$ to $\$ 788$, respectively. The genetically targeted IDDM prediction strategy is remarkably cost-saving as compared with the pure immunological strategy mainly because fewer subjects will need retesting during the follow-up. [Diabetologia (1998) 41: 79--85]

Keywords Insulin-dependent diabetes mellitus, prediction, prevention, screening, genetic risk, islet cell antibodies, autoimmunity, costs.
Prevention of insulin-dependent diabetes mellitus (IDDM) and possibly other multifactorial, multigenic diseases may soon become a reality [1--5]. IDDM is an excellent candidate for prevention due to the high incidence of the disease in the western world and the sizeable life-long costs associated with the disease. Furthermore, IDDM susceptibility is geneti-

Received: 30 April 1997 and in final revised form: 27 August 1997

Corresponding author: J. Hahl, M.Sc.Economics, University of Turku, MediCity Research Laboratory, Tykistökatu 6 A, 20520 Turku, Finland

Abbreviations: IDDM, Insulin-dependent diabetes mellitus; ICA, islet cell autoantibodies; PCR, polymerase chain reaction. cally determined [6,7], and the clinical disease is preceded by an asymptomatic latency period characterised by the presence of markers of islet cell autoimmunity. These markers associate closely with progressive destruction of the insulin-producing beta cells in the pancreatic islets of Langerhans, and the destruction may ultimately lead to clinical IDDM.

There are numerous options for disease prevention in animal models of autoimmune diabetes, suggesting that some treatments may also be effective in man [1]. Studies have been launched recently to predict IDDM (e.g. the DAISY project) [8--16] or to prevent clinical disease as well (e.g. the American DPT-1 trial, the European ENDIT study $[17,18]$ and the Finnish DIPP project, see below), and several trials are in the planning phase. As only about $10 \%$ of new 
IDDM cases occur in families with previously affected subjects, subjects with increased IDDM risk have to be found from the general population. Accordingly, selection of the most cost-efficient screening approach becomes a critical issue for the payer of the service. As seroconversion to autoantibody positivity may occur at any age, the strategy of predicting IDDM may be based on: 1) repeated analyses of marker(s) of disease-associated autoimmunity in the entire population (the pure immunological strategy); or 2) initial evaluation of genetic IDDM susceptibility [7], followed by repeated measurement of the autoimmune markers only in the smaller, susceptibility restricted population (the genetically targeted strategy).

The two prediction strategies are compared using economic data collected in the Finnish populationbased IDDM Prediction and Prevention Project (DIPP). In the DIPP project HLA-DQB1 allele combinations tightly linked with IDDM susceptibility or protection have been analysed in over 11000 consecutive cord blood samples. Islet-specific autoantibodies, primarily islet cell autoantibodies (ICA), have then been measured at defined intervals in subjects at increased genetic risk for IDDM. Data on the expenses accrued during the first 2 years of the DIPP trial provide detailed information for comparison of the costs caused by the two IDDM prediction strategies. Sensitivity analysis of the factors which affect cost accumulation shows that marked variations in disease incidence, prevalence of autoimmune markers or in other contributing factors have a minimal influence on the outcome of the comparison.

\section{Subjects and methods}

Design of the DIPP trial. The population-based DIPP trial, launched in Turku and Oulu in Finland in 1994 and 1995, respectively, is based on screening of HLA-DQB1 risk alleles in the cord blood of all newborn infants (8500 annual births), subsequent immunological follow-up of those who turn out to be at increased genetic risk, and identification of the time of onset of islet-specific autoimmunity. Finally, those who are both genetically and immunologically at increased risk are randomised to receive nasal insulin or placebo in a double-blind preventive treatment trial. Genetic screening, as applied in the DIPP trial, identifies $60--80 \%$ of those who will develop IDDM in Finland before the age of 15 years [7].

In February 1997, 11721 consecutive cord blood samples had been screened for the designated HLA-DQB1 alleles after informed consent by the parents. Blood was dried on filter paper and the alleles were determined using PCR, lanthanide chelate labelled oligonucleotide probes and time-resolved detection of the label [19]. Of those screened, $13.0 \%$ carry the risk allele combinations $* 0302 / * 0201$ or $* 0302 / * \mathrm{x}(* \mathrm{x}=\mathrm{a}$ neutral allele) without protective alleles *0602, *0603 or *0301. Those at increased genetic risk (propositi) and their at-risk siblings are followed for development of islet autoimmunity at 3month intervals until 2 years of age and biannually thereafter. As the oldest trial propositi are now aged slightly over 2 years,
Table 1. Valuation of different cost items

\begin{tabular}{|c|c|}
\hline Cost item & Valuation \\
\hline \multicolumn{2}{|l|}{ Variable costs } \\
\hline $\begin{array}{l}\text { Wages of personnel } \\
\text { (including payroll fringe } \\
\text { costs) }\end{array}$ & $\begin{array}{l}\text { Average wage rates. Length of each } \\
\text { work phase is based on actual time } \\
\text { used in the procedure. Payroll fringe } \\
\text { costs are estimated to be } 47 \%\end{array}$ \\
\hline $\begin{array}{l}\text { Laboratory equipment } \\
\text { and reagents }\end{array}$ & Market prices \\
\hline Medical and other supplies & Market prices \\
\hline $\begin{array}{l}\text { Office expenditure } \\
\text { (materials, mail, phone etc.) }\end{array}$ & Market prices \\
\hline Sample transportation & $\begin{array}{l}\text { As the service would have been } \\
\text { bought from outside the hospital; } \\
\text { market prices }\end{array}$ \\
\hline \multicolumn{2}{|l|}{ Fixed costs } \\
\hline Overheads & $\begin{array}{l}65 \% \text { overhead rate. This rate is used } \\
\text { by the hospital accounting in allo- } \\
\text { cating fixed costs (administration, } \\
\text { maintenance etc.) to outpatient care } \\
\text { at the Department of Paediatrics, } \\
\text { Turku University Hospital }\end{array}$ \\
\hline
\end{tabular}

there are limited data on the rate and age distribution of seroconversion to positivity for disease-associated autoantibodies.

In the following calculations the at-risk children are assumed to comprise $13.0 \%$ of the population as suggested by the DIPP trial. Of those at increased genetic risk, $23.5 \%$ are expected to have a risk of $7 \%(* 0302 / * 0201)$ and $76.5 \%$ a risk of $3 \%(* 0302 / * x)$ of developing IDDM before 15 years of age [7]. Consequently, the average risk of the targeted population is $4 \%$, while the risk in the background population is $0.66 \%$.

About 3--4\% of Finnish school-aged children test positive for ICA [8]. This high proportion is probably closely associated with the high IDDM incidence in Finland [20,21], as the prevalence of ICA positivity appears to be tightly linked with IDDM incidence in childhood [22--25]. New ICA positive subjects are assumed to accumulate at a constant rate, as the proportions of ICA positive children in different age categories are not available. If $50 \%$ of the children, who are genetically at increased risk and have ICA, develop IDDM before 15 years of age, $8 \%$ of the children who are genetically at increased risk should test positive for ICA by 15 years of age. Assuming a stable seroconversion rate through childhood allows us to simplify the calculations and use average numbers of ICA positive children in the analysis.

The intra- and inter-assay variabilities of the autoimmunity marker tests have been defined [26]. The laboratory has repeatedly participated in the international ICA standardisation workshops, and achieved sensitivity and specificity figures $98 \%$ and greater.

Cost analysis. In this analysis relevant costs were identified, quantified by natural units (hours of physician's time, reagent consumption etc.), and valued in commensurable monetary units $[27,28]$. Since the financial burden to the health care system caused by IDDM prediction was studied [29,30], the provider's viewpoint was chosen. Average costing was used as large populations were studied and the frame of reference was the aggregate [31]. Consequently, indirect [32] and intangible [33] costs were excluded from the analysis [30]. Meanwhile, costs such as payroll fringe costs were taken into account, even though they are transfers by nature. 
For most direct cost items market prices were available (Table 1). Analysis of fixed costs was more complicated, as allocation of costs to different procedures was difficult, although market values for larger entities were available. Thus, fixed costs were allocated using overhead rates defined by the hospital accounting in Turku University Hospital [30].

Direct costs. Average wages in Finland were used [34] (Table 2). Time spent at different phases of the two prediction protocols was analysed accurately step by step assuming that the labour capacity was in full use (Table 2).

Direct costs in the genetically targeted strategy accrue from: 1) genetic screening, 2) ICA follow-up and 3) counselling of the families (Table 2). The expenses of genetic screening include costs of recruitment and signing of the informed consent, drawing and handling of the blood sample, sample transports, laboratory reagents and disposables, data handling and reporting, office expenditures, first family counselling after screening as well as overhead costs. All wages and payroll fringe costs caused by these events are also included. These costs accrue only once at birth.

The expenses of autoimmunity follow-up recur and cover costs of drawing the blood sample, sample preparation, transportation, autoimmunity screening, laboratory equipment and reagents, supplies, data handling and reporting, office expenditures and overheads. All associated wages and payroll fringe costs are also included. Costs of counselling when the child has a blood sample drawn include wages of the counsellors (paediatric resident, paediatric nurse) with payroll fringe costs and overheads.

Direct costs accruing when the pure immunological prediction strategy is used comprise costs of obtaining, preparing and transporting the sample, testing for autoimmune marker(s), laboratory equipment and reagents, supplies, reporting, office expenditures and overheads as well as the wages of the personnel with payroll fringe costs.

Autoimmune markers (presently ICA in the DIPP project) are measured at birth, then at 3-month intervals, and biannually after 2 years of age (Fig. 1). Samples are drawn more frequently during the first 2 years of life, as pre-IDDM might progress to clinical IDDM more aggressively in young children, and preventive treatment thus should probably be initiated soon after seroconversion. Prolongation of the sampling intervals would decrease costs of both prediction strategies but would have a minimal influence on the relative costs of the two strategies. As accurate data of the timing of autoimmune seroconversions are missing, we excluded counselling expenses from the costs of the pure immunological strategy. As counselling contributes substantially to the costs, the true costs of the pure immunological approach would be higher than shown in this study.

\section{Results}

Direct costs of genetically targeted IDDM prediction. Direct costs of HLA-DQB1 risk allele analysis including all accompanying costs (as defined in Methods) are US\$ 127 (US\$ 1 = Finnmark 4.90, February 1997) for a child who is genetically at increased risk, whereas the costs are $\$ 68$ for a non-risk child. The difference in costs is due to the expenses caused by counselling of those who are at increased risk. Since $13.0 \%$ of children are genetically at increased risk,
Table 2. Per sample working times and average monthly wages of personnel in different phases of genetic screening, ICA follow-up and counselling

\begin{tabular}{llc}
\hline Phase & $\begin{array}{l}\text { Personnel } \\
\text { (wage/month \$) }\end{array}$ & $\begin{array}{c}\text { Time } \\
\text { (min) }\end{array}$ \\
\hline $\begin{array}{l}\text { Genetic screening } \\
\text { Recruitment }\end{array}$ & Paediatric nurse (2156) & 30 \\
Sample drawing & Paediatric nurse & 10 \\
Screening & Laboratory nurse (2019) & 35 \\
& Laboratory technician (2449) & 15 \\
Office employee (1649) & 5 \\
Reporting & Paediatric nurse & $5--15$ \\
Counselling & Paediatrician (3594) (resident) & 45 \\
& Paediatric nurse & 45 \\
ICA follow-up & & \\
Sample drawing & Laboratory nurse & $15--30$ \\
and handling & & \\
Screening & Laboratory nurse & 15 or \\
(ICA - or + ) & & 105 \\
Counselling & & \\
3-monthly sessions & Paediatric nurse & 45 \\
Other sessions & Paediatric nurse & 30 \\
Once per year & Paediatrician & 30 \\
sessions & Paediatric nurse & 30 \\
\hline
\end{tabular}

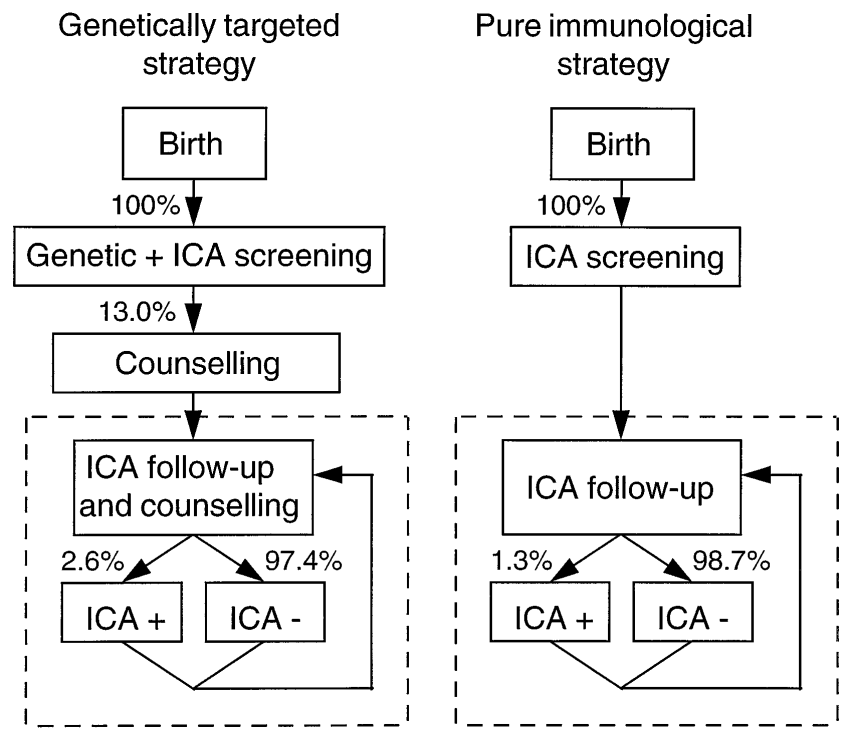

Fig. 1. Diagram of the deterministic model used in the cost calculations. The percentages in the diagram show true or expected proportions of the children progressing through the pathway (follow-up time 10 years). The areas inside the dashed boxes are executed four times per year in the first 2 years of follow-up and then semiannually. As described in detail in Methods, the cost calculations in the pure immunological strategy contain no counselling costs

the average costs of screening are $\$ 76$ per child, while the costs of finding one at-risk child are $\$ 585$.

The costs of collecting and analysing ICA positive and negative follow-up samples are $\$ 73$ and $\$ 29$, respectively, since the titration of the positive samples 
demands extra work. The costs of the cord blood ICA measurement are only $\$ 61$ and $\$ 17$ for ICA positive and ICA negative samples, as the handling costs of the cord blood are included in the costs of genetic screening.

The first counselling visit in the DIPP trial for families with a child carrying increased genetic risk is 4 to 8 weeks after delivery. A physician and a research nurse tell the parents about diabetes and the DIPP trial and clarify what their child's IDDM risk means in practical terms. The three-monthly visits before 2 years of age and biannual visits thereafter are covered by the nurse alone. A physician, assisted by a nurse, meets the family once a year. Blood samples are drawn by a laboratory nurse at every follow-up visit. The direct costs caused by the initial counselling visit, various visits to the nurse, and the annual visits to see the physician as well as the nurse are $\$ 54$, \$16--\$23, and \$36, respectively.

The costs caused by a genetically at-risk child who is continuously ICA negative are \$783, \$ 198 and \$ 109 in the first, second and later years, respectively; those of an ICA positive child are $\$ 960, \$ 376$ and \$198 (Fig. 2).

Using average direct costs of genetic screening, ICA follow-up and counselling, the 10-year direct costs of the trial are $\$ 245$ per child, or $\$ 1885$ per child who is genetically at risk. At present values using a $5 \%$ discount rate, 10 -year costs are $\$ 217$ per child.

Direct costs of the pure immunological strategy, i.e. repeated screening of the entire population for ICA. If marker(s) of autoimmunity are measured repeatedly in the general population without previous genetic risk assessment, the number of children to be studied remains unchanged. As children in the model used are studied more frequently in the first 2 years of life, the costs accrue faster at the beginning than later. The costs of the pure immunological strategy are $\$$ $145, \$ 118$ and $\$ 59$ in the first, second and the following years, respectively (Fig. 3). The direct 10-year costs are thus $\$ 733$ per child (present value, $5 \%$ discounting, \$ 619).

Sensitivity analysis. Sensitivity analysis shows that neither the uncertainties caused by the unconfirmed proportions of the genetically at-risk children and of children positive for autoimmune markers nor the choice of the discount rate affects the main finding, i.e. the genetically targeted prediction programme is extremely cost saving as compared with repeated autoantibody screening of the entire population (Table 3). Increases or decreases in the working time used for the various phases of risk assessment fail to influence this outcome. In the extreme case $1(\mathrm{Ta}-$ ble 3), when all values maximally favour the immunological strategy, its discounted costs exceed those caused by the targeted strategy by close to $80 \%$

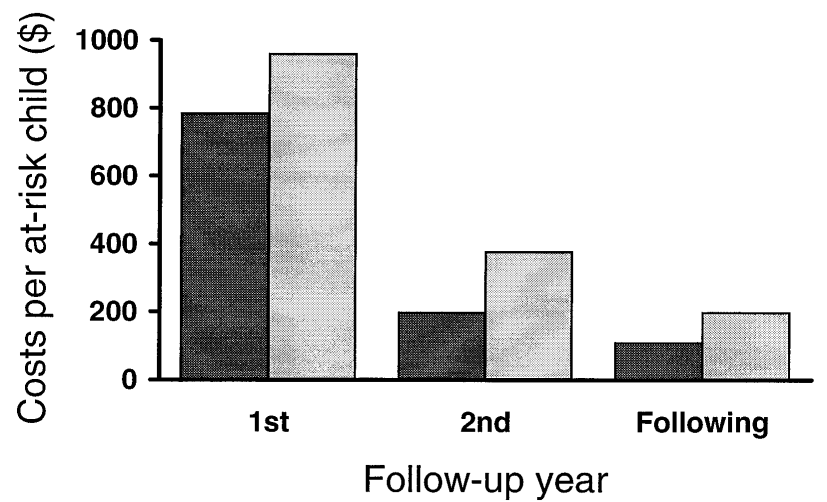

Fig. 2. Annual direct costs of IDDM prediction based on the analysis of genetic IDDM risk at birth combined with ICA follow-up and counselling of families with children at increased genetic risk. Costs differ slightly between ICA negative (I) and ICA positive (II) children due to the need of titration for the ICA positive samples

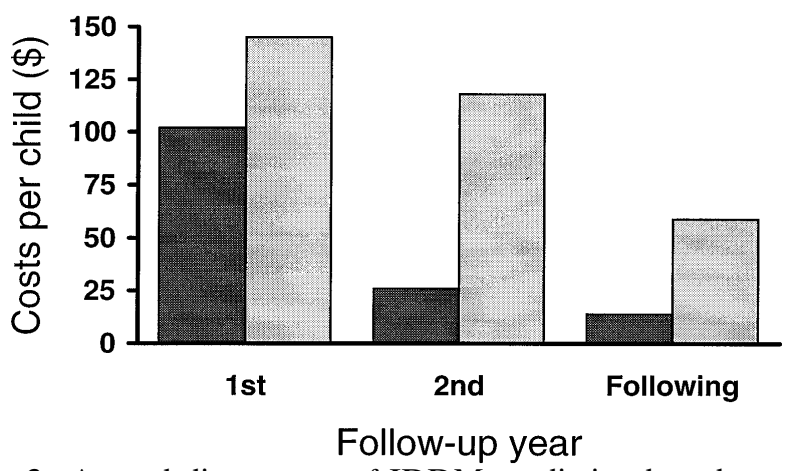

Fig. 3. Annual direct costs of IDDM prediction based on: 1) the analysis of genetic IDDM risk at birth, ICA follow-up and counselling of families with children who are at increased genetic risk (genetically targeted strategy) (I) ; or 2) on repeated ICA follow-up of the entire population (pure immunological strategy) (1]). Cost calculations in (2) contain no costs for counselling (see Methods). Inclusion of counselling costs would further increase the cost difference between the two strategies

(\$430 vs \$241). If the assumptions favour the targeted approach (extreme case 2, Table 3), the present value of the 10-year costs of the genetically targeted prediction (\$152) are only $19 \%$ of those of the pure immunological programme (\$788), even though the costs of the immunological strategy contain no counselling (see Methods).

\section{Discussion}

Accurate data on the expenses accruing in the DIPP project, where over 11000 consecutive newborn infants have been screened for genetic risk of IDDM and those at increased genetic risk have been immunologically followed up, have now been used as background for a comparison of the economical implica- 
Table 3. Sensitivity analysis of present values of 10 -year total direct costs per child

\begin{tabular}{|c|c|c|}
\hline $\begin{array}{l}\text { Variables } \\
\text { (Empirical finding or basic } \\
\text { assumption in parenthesis) }\end{array}$ & $\begin{array}{l}\text { Genetically } \\
\text { targeted } \\
\text { strategy }(\$)\end{array}$ & $\begin{array}{l}\text { Pure immu- } \\
\text { nological } \\
\text { strategy }(\$)\end{array}$ \\
\hline Baseline case & 217 & 619 \\
\hline Genetic risk $8 \%(13.0 \%)$ & 160 & 619 \\
\hline Genetic risk $16 \%(13.0 \%)$ & 251 & 619 \\
\hline $\begin{array}{l}\text { ICA positivity of risk children } \\
10 \%(8 \%)\end{array}$ & 218 & 619 \\
\hline $\begin{array}{l}\text { ICA positivity of unselected } \\
\text { population } 2 \%(4 \%)\end{array}$ & 217 & 613 \\
\hline Work time of each phase $+25 \%$ & 262 & 743 \\
\hline Work time of each phase $-25 \%$ & 171 & 495 \\
\hline Discount rate $3 \%$ (5\%) & 227 & 660 \\
\hline Discount rate $10 \%(5 \%)$ & 197 & 537 \\
\hline $\begin{array}{l}\text { Extreme case } 1 \text { : } \\
\text { genetic risk } 16 \% \text {, ICA + of at-risk } \\
\text { children } 10 \% \text { and of unselected } \\
\text { population } 2 \% \text {, work time in } \\
\text { screening and counselling }+25 \% \\
\text { and in ICA follow-up }-25 \%, \\
\text { discount rate } 10 \%\end{array}$ & 241 & 430 \\
\hline $\begin{array}{l}\text { Extreme case } 2 \text { : } \\
\text { genetic risk } 8 \% \text {, work time in } \\
\text { screening and counselling - }-25 \% \\
\text { and in ICA follow-up }+25 \%, \\
\text { discount rate } 3 \%\end{array}$ & 152 & 788 \\
\hline
\end{tabular}

tions of two IDDM prediction strategies. For the genetically targeted strategy the 10-year investment needed is $\$ 217$ per child (present value, $5 \%$ discounting), i.e. the costs are markedly lower than those caused by the pure immunological approach, which relies on repeated immunological testing of the entire population.

As the effectiveness of the currently tested and proposed preventive treatments remain unknown and the life-time costs of IDDM have only been estimated roughly, cost-effectiveness analysis of IDDM prediction and prevention is not yet achievable. However, some estimations of the required efficacy of the prevention are possible. In the United States, the direct costs of diabetes (IDDM and non-insulin-dependent diabetes, NIDDM) are estimated to be $\$ 90$ billion per year [35] or $\$ 12500$ per patient with diabetes. These figures are adopted here as the best estimate of the costs of IDDM. Simplisticly, delay of clinical IDDM by one single year accounts in health care expenditures for a saving of $\$ 12500$ per person. As the annual average direct costs of the genetically targeted IDDM prediction strategy are \$ 189 (i.e. 10year direct costs $\$ 1885$ divided by 10 years) per atrisk child, and the risk of the at-risk children to have IDDM is $4 \%$, the costs of finding one at-risk child who without prevention will develop IDDM are $\$ 4725$ per year. Thus, an average delay of IDDM by 0.38 years per all years the immunological follow-up has been continued would cover the costs of the population-based IDDM prediction $(\$ 12500 \times 0.38=$ $\$ 4750$ ). Thus, if a child has been observed for 8 years when seroconversion to positivity for the autoimmune marker(s) occurs, the preventive treatment should delay the manifestation of the clinical disease by 3 years ( 8 years $\mathrm{x} 0.38=3.0$ years) to cover the costs of the prediction.

Of the two strategies compared in this study, the genetically targeted IDDM prediction is clearly costsaving as compared with repeated ICA screening of the entire population. The differences in costs are sizeable in the long run, as seroconversion to autoantibody positivity may occur at any age, and follow-up thus has to be continued at least throughout childhood. As some of the means which are currently tested for IDDM prevention are probably able to at least delay the onset of clinical IDDM, and the development of more efficient preventive modalities is in progress, selection of feasible, cost-efficient means of recognizing the onset of the disease process(es) combined with early preventive measures are of high potential value. The health economic impact of prediction and prevention programmes also depends on the age at disease manifestation in the population. Finland, which has an exceptionally high IDDM incidence in childhood and where the mean age at diagnosis is low, will thus gain relatively more than other countries with a frequent screening schedule.

The number of children who will need follow-up is critical for the economical outcome in the cost comparison of the prediction strategies. Genetic screening excludes $87 \%$ of the population from the followup; such exclusion is the key issue in cost reduction. However, the sensitivities of the two strategies differ markedly. As genetic screening identifies only 60-$80 \%$ of those who will develop IDDM and the capability of ICA monitoring to find those who will develop IDDM is $70--90 \%$, the genetically targeted method will find only $42--72 \%$ of those who will progress to clinical IDDM. Thus, the cost-savings due to the genetically targeted strategy should be balanced with the number of subjects with "unprevented" " IDDM. On the other hand, the number of "unprevented" "IDDM cases depends on the effectiveness of the preventive treatment, i.e. what proportion of the IDDM cases occurring in the population ruled out from the follow-up is actually preventable using the prevention therapy selected. Another factor which may influence the sensitivities of the two strategies is the fact that the proportion of ICA positive children who actually are going to develop IDDM is probably larger among the ICA positive, genetically at-risk children than among the ICA positive children in the general population, as recently suggested by the ICARUS study [36]. Since marked improvements in the predictive sensitivity of disease-specific autoimmune markers are unlikely (best combination 
sensitivities now exceed $90 \%$ ), whereas additional IDDM-associated genetic markers will probably be characterized [37,38], genetically targeted IDDM prediction will be increasingly favoured as the first screening measure. As preventive measures for other multifactorial diseases are being developed, the same samples collected for genetic IDDM prediction will be available also for other disease-targeted predictive measures. Improved genetic methodologies and the growing number of samples analysed will likely increase analytic efficiency and cut down sample costs.

In conclusion, this study shows that in IDDM prediction the genetically targeted strategy together with autoantibody follow-up of those who are at increased genetic risk is extremely cost-saving as compared with the pure immunological approach, i.e. repeated immunological screening of the entire population. The costs of the targeted strategy are smaller, even though costs of counselling were excluded from the expenses of the pure immunological strategy. The cost difference between the two strategies is of such a magnitude that it is likely to be decisive in the selection of IDDM prediction strategies in all western countries. The genetically targeted strategy will probably also improve follow-up compliance in populations with limited knowledge of the burden caused by IDDM, and may allow individualization of the follow-up practices and preventive therapy according to the child's inherited risk category.

Acknowledgements. This study has been supported by the Yrjö Jahnsson Foundation, the Oskar Öflund Foundation, the Finnish Office for Health Care Technology Assessment (STAKES/ FinOHTA), and the Diabetes Research Foundation, Finland.

\section{References}

1. Bowman MA, Leiter EH, Atkinson MA (1994) Prevention of diabetes in the NOD mouse: implications for therapeutic intervention in human disease. Immunol Today 15: 115-120

2. Elias D, Cohen IR (1994) Peptide therapy for diabetes in NOD mice. Lancet 343: 704--706

3. Keller RJ, Eisenbarth GS, Jackson RA (1993) Insulin prophylaxis in individuals at high risk of type I diabetes. Lancet 341: 1291

4. Lapinleimu H, Viikari J, Jokinen E et al. (1995) Prospective randomised trial in 1062 infants of diet low saturated fat and cholesterol. Lancet 345: 471--476

5. Niinikoski H, Viikari J, Rönnemaa T et al. (1996) Prospective randomized trial of low-saturated-fat, low-cholesterol diet during the first 3 years of life: The STRIP Baby Project. Circulation 94: 1386--1393

6. Becker D, Cakan N, LaPorte R, Drash A, Trucco M (1995) High risk DG alleles improve IDDM prediction in $\mathrm{ICA}+$ ve first degree relatives with decreased insulin secretion. Autoimmunity 21: 60 (Abstract)
7. Reijonen H, Ilonen J, Knip M, A< kerblom H (1991) HLA-DQB1 alleles and absence of Asp 57 as susceptibility factors of IDDM in Finland. Diabetes 40: 1640--1644

8. Karjalainen JK (1990) Islet cell antibodies as predictive markers for IDDM in children with high background incidence of disease. Diabetes 39: 1144--1150

9. Bazzigaluppi E, Pastore MR, Bonfanti R et al. (1995) GAD antibodies as a primary screening marker in first degree relatives of IDDM patients. Autoimmunity 21: 62 (Abstract)

10. Behme MT, Leushner J (1995) Predictive markers in first degree relatives of patients with insulin dependent diabetes mellitus. Autoimmunity 21: 62 (Abstract)

11. Karjalainen J, Vähäsalo $\mathrm{P}, \mathrm{A}<$ kerblom HK, Knip M, and the DiMe-Study Group (1995) Predictive value of islet cell and insulin autoantibodies for IDDM in siblings less than 20 years of age is not associated with age at initial detection of autoantibodies. Autoimmunity 21: 65 (Abstract)

12. Kulmala P, Petersen JS, Vähäsalo P et al. (1995) Life-table analysis of progression to diabetes in siblings of children with IDDM in relation to IDDM associated autoantibodies. Autoimmunity 21: 61 (Abstract)

13. Leech NJ, McCulloch DK, Rowe RE, Palmer JP, Hagopian WA (1995) The role of GAD65AB in the prediction of IDDM in the general population and it's relationship to other susceptibility markers. Autoimmunity 21: 63 (Abstract)

14. Mackay IR, Byron SL, Rowley MJ, Whittingham SF, Zimmet PZ, Tuomilehto J (1995) Islet cell antibodies and antiGAD as predictors of diabetes. Autoimmunity 21: 64 (Abstract)

15. Morales J, Puig-Domingo M, Vinets I, Ronningen KS, Dyrberg T, de Leiva A (1995) IDDM risk assesment of first degree relatives of a Spanish cohort by using a multiantibody screening programme combined with HLA markers. Autoimmunity 21: 61 (Abstract)

16. Verge CF, Gianani R, Yu L et al. (1995) Prediction of IDDM using a combination of insulin, glutamic acid decarboxylase and ICA512 autoantibodies. Autoimmunity 21: 59 (Abstract)

17. The DPT-1 Study Group (1995) The diabetes prevention trial -- type 1 diabetes (DPT-1): Progress report. Autoimmunity 21: 66 (Abstract)

18. Reimers JI, Andersen HU, Pociot F (1994) Nicotinamide and prevention of insulin-dependent diabetes mellitus. Rationale, effects, toxicology and clinical experiences. ENDIT Group. Ugeskr Laeger 156: 461--465

19. Sjöroos M, Iitiä A, Ilonen J, Reijonen H, Lövgren T (1995) Triple-label hybridization assay for type-1 diabetes-related HLA alleles. Biotechniques 18: 870--877

20. Kangas T (1995) The FINNDIAB report. Health care of people with diabetes in Finland. Research Reports 58. Stakes, Saarijärvi

21. Simell TT, Sintonen H, Hahl J, Simell OG (1996) Costs of insulin-dependent diabetes mellitus. PharmacoEconomics 9: $24--38$

22. Schiffrin A, Ciampi A, Hendricks L, Rozen R, Weitzner G (1994) Evidence for different clinical subtypes of type 1 diabetes mellitus: a prospective study. Diabetes Res Clin Pract 23: 95--102

23. Schiffrin A, Colle E, Ciampi A, Hendricks L, Poussier P (1993) Different rates of conversion to IDDM in siblings of type 1 diabetic children: the Montreal family study. Diabetes Res Clin Pract 21: 1--3

24. Bonifacio E, Genovese S, Braghi S et al. (1995) Islet autoantibody markers in IDDM: risk assessment strategies yielding high sensitivity. Diabetologia 38: 816--822 
25. Bingley PJ, Christie MR, Bonifacio E et al. (1994) Combined analysis of autoantibodies improves prediction of IDDM in islet cell antibody-positive relatives. Diabetes 43: $1304--1310$

26. Mustonen A, Knip M, Huttunen NP, Puukka R, Käär M, A $<$ kerblom HK (1984) Evidence of delayed beta-cell destruction in type 1 (insulin dependent) diabetic patients with persisting complement-fixing cytoplasmic islet cell antibodies. Diabetologia 27: 421--426

27. Drummond MF (1980) Principles of economic appraisal in health care. Oxford University Press, Oxford

28. Robinson R (1993) Economic evaluation and health care. Costs and cost-minimisation analysis. BMJ 307: 726--728

29. Luce RB, Elixhauser A (1990) Estimating costs in the economic evaluation of medical technologies. Int $\mathrm{J}$ Technol Assess Health Care 6: 57--75
30. Weinstein MC (1990) Principles of cost-effective resource allocation in health care organizations. Int $\mathrm{J}$ Technol Assess Health Care 6: 93--103

31. Whynes DK, Walker AR (1995) On approximations in treatment costing. Health Econ 4: 31--39

32. Koopmanschap MA, Rutten FFH (1994) The impact of indirect costs on outcomes of health care programs. Health Econ 3: 385--393

33. Drummond MF, Stoddart GL, Torrance GW (1987) Methods for the economic evaluation of health care programmes. Oxford University Press, Oxford

34. Official Statistics of Finland (1994) Wage statistics 1993/ 1994. Wages. Statistics Center of Finland, Helsinki

35. Department of Health and Human Services (1995) Disease-specific estimates of direct and indirect costs of illness and NIH support. Public Health Service. National Institutes of Health. Office of the Director

36. Bingley PJ, Colman P, Eisenbarth GS et al. (1992) Standardization of IVGTT to predict IDDM. Diabetes Care 15: $1313--1316$

37. Schuler GD, Boguski MS, Stewart EA et al. (1996) A gene map of the human genome. Science 274: 540

38. Marshall E, Pennisi E (1996) NIH launches the final push to sequence the genome. Science 272: 188--189 